\title{
Renal biopsy in a general hospital
}

\author{
PAUL TURNER ${ }^{1}$
}

From Edgware General Hospital, Middlesex

SYNOPSIS The results of 46 attempted renal biopsies on 44 patients in a general hospital are discussed.

The diagnostic value of this procedure is the subject of controversy in the literature. While it may be considerable if carried out in specialist units, the results in this series suggest that renal biopsy is usually only confirmatory and that careful clinical and laboratory assessment provides a reasonable diagnosis in most cases.

Percutaneous renal biopsy is an established diagnostic procedure. Iversen and Brun (1951) and Brun (1954) described a technique of aspiration biopsy using a serrated needle with patients sitting in an upright position. Their success was not repeated by other workers (Parrish and Howe, 1953) who found that this position was unsatisfactory because of renal mobility and the patients' tendency to faintness. Kark and Muehrcke (1954) described renal biopsy in the prone position, locating the kidney with an exploring needle and then obtaining a specimen of tissue with a Vim-Silverman needle. Since that time, many investigators have reported large series of biopsies obtained by this method. More recently Kerr (1960) introduced the modified Menghini needle in place of the Vim-Silverman, and suggested that it had the advantage not only of being easier to manipulate but of obtaining more glomeruli than the Vim-Silverman needle.

\section{MATERIAL}

Forty-four patients were referred for renal biopsy. They could be classified broadly into the following groups of conditions for investigation:-

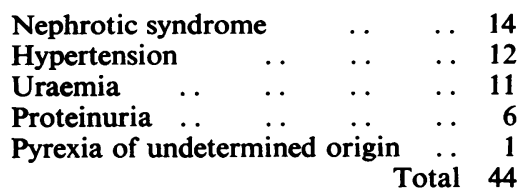

There were 20 males and 24 females, and their ages ranged from 14 to 72 years with a mean of 49 years. One patient was comatose at the time of biopsy; the rest were conscious and cooperative.

'Present address: Department of Pharmacology, St. Bartholomew's Hospital Medical College, London, E.C.1.

Received for publication 6 March 1963.
METHOD

Estimations of prothrombin time and platelet count were carried out before biopsy, and a pint of blood was crossmatched. The latter was never required for use. A plain radiograph of the abdomen was obtained from each patient, and where possible an intravenous pyelogram $\vec{\bullet}$ was performed in order to localize the kidney shadows. $\mathrm{E}$ The patient was sedated with quinalbarbitone, $100 \mathrm{mg}$., half an hour before the procedure. Biopsy was performed by the percutaneous route using a modified Menghini needle.

The patient was placed in a prone position with the hips flexed over pillows and a sand-bag. Surface markings $\stackrel{\mathbb{D}}{\triangle}$ were then drawn on the skin to show the mid-line along $\overrightarrow{\vec{A}}$ the spinous processes of the lumbar vertebrae, the lower border of the twelfth rib, the upper border of the iliac crest, and the outer border by palpation of the sacrospinalis muscle (Fig. 1). The site of puncture was in the angle between the twelfth rib and the outer border of the sacrospinalis muscle on the left side. After achieving local anaesthesia, the depth and direction of the left $\exists$ kidney was determined with a fine probing needle. It was considered to have entered the kidney or its capsule when it swung widely and paradoxically as the patient breathed deeply. The biopsy needle was then introduced to a depth 옥 considered to be just outside the capsule and emptied of $>$ tissue by flushing it through with a small quantity of saline. The patient was asked to strain down as though for defaecation in order to contract the muscles of the $\Omega$ anterior abdominal wall and thus push the kidneys back- $N$ wards against the posterior wall. The needle was then N introduced into the kidney, with simultaneous aspiration, slightly rotated and withdrawn.

The specimen obtained was gently flushed from the needle with saline. In the first few attempts it was examined on a piece of filter paper, and a portion was cut off for $:-$ culture before the larger part was fixed. Culture was con- $\underline{T}$ sistently unrewarding, however, and it was felt that rather than delay fixation the specimen should be flushed directly into fixative. This technique was used, therefore, in the large majority of cases. 

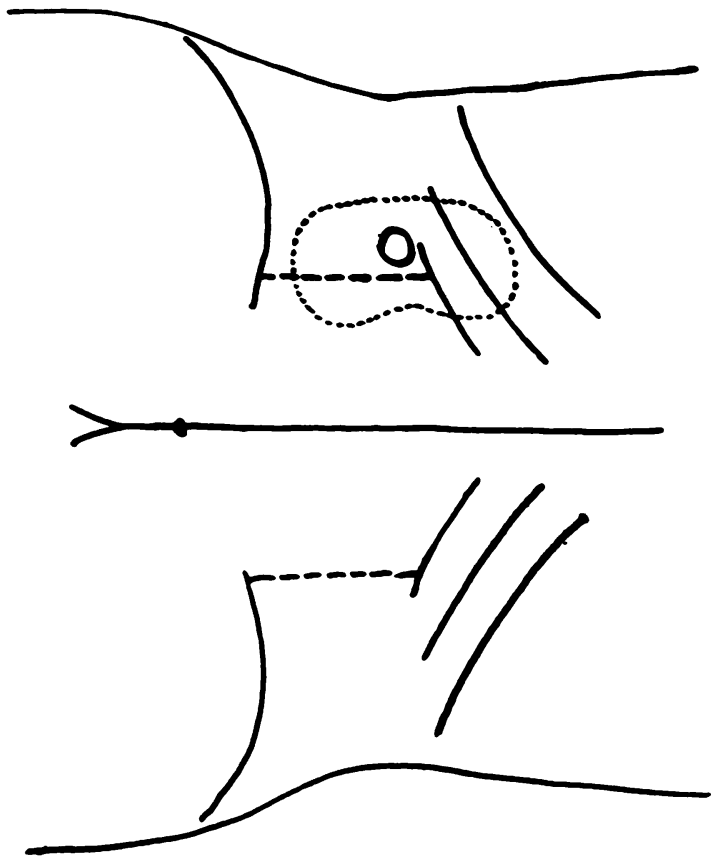

FIG. 1. Surface markings for renal biopsy.

Two hours after the biopsy, a mid-stream specimen of urine was obtained in 20 patients and examined for the presence of red cells and organisms and cultured.

The specimen was fixed in $10 \%$ formal saline. Sections were cut at $3 \mu$ thickness and usually stained with haematoxylin and eosin. They were not cut and studied serially but further sections were cut if other stains were required.

\section{RESULTS}

In 42 patients renal biopsy was carried out on only one occasion, but in two patients it was repeated. The first of these presented with the nephrotic syndrome, and although the initial biopsy had five glomeruli for study and seemed adequate histologically, no abnormality was detected. The second biopsy contained 15 glomeruli, but still no abnormality was found. In the other patient, who also had the nephrotic syndrome, no glomeruli were obtained in the first specimen, but on the second attempt six were present and the characteristic histological changes of Ellis type 2 nephritis were found.

A total of 46 attempts, therefore, was made; of these, 39 were successful in obtaining renal tissue and seven failed. The number of glomeruli per specimen varied from 0 to 54 with a mean of 10 and are given in more detail in Table $\mathrm{I}$.

In four patients it was possible to make a histological diagnosis on the basis of less than five glomeruli (Table II), while in six specimens containing more than five glomeruli, and three of these with

TABLE I

NUMBER OF GLOMERULI PER SPECIMEN

Number of Glomeruli

Number of Biopsies

\begin{tabular}{cc} 
Number of Glomeruli & Number of Biopsies \\
\hline $0-4$ & 10 \\
$5-9$ & 14 \\
$10-14$ & 4 \\
$15-19$ & 5 \\
$20-24$ & 1 \\
$25-29$ & 3 \\
$30-39$ & 1 \\
$40-49$ & 0 \\
$50-59$ & 1
\end{tabular}

TABLE II

HISTOLOGICAL DIAGNOSIS RELATED TO NO. OF GLOMERULI Histological Diagnosis Number of Glomeruli

Chronic glomerulonephritis

Diabetic intercapillary glomerulosclerosis

Diabetic intercapillary glomerulosclerosis

Acute glomerulonephritis

TABLE III

RESULT OF HISTOLOGICAL EXAMINATION

\begin{tabular}{lclc}
$\begin{array}{l}\text { Condition } \\
\text { Investigated }\end{array}$ & $\begin{array}{l}\text { No. of } \\
\text { Patients }\end{array}$ & Histological Diagnosis & $\begin{array}{c}\text { No. of } \\
\text { Patients }\end{array}$ \\
\hline Nephrotic syndrome & 12 & $\begin{array}{l}\text { Diabetic intercapillary } \\
\text { glomerulosclerosis } \\
\text { Ellis type 2 nephritis }\end{array}$ & 5 \\
& & $\begin{array}{l}\text { Amyloidosis } \\
\text { Renal vein thrombosis } \\
\text { N.A.D. (rectal biopsy } \\
\text { showed amyloid in one) }\end{array}$ & 1 \\
& & 3
\end{tabular}

Hypertension

11 Benign nephrosclerosis

Malignant nephrosclerosis $\quad \mathbf{8}^{\mathbf{1}}$

Chronic pyelonephritis Systemic lupus erythematosus 1

Not made

Uraemia

Proteinuria

Pyrexia of undetermined origin

${ }^{1}$ Two later shown at necropsy to have polycystic kidneys.

more than 10 , no histological diagnosis could be made. The results of histological examination in the various groups of patients are set out in Table III.

It can be seen that no histological diagnosis was made in nine cases. In only one patient did the histology vary significantly from the clinical diagnosis. This was in a man of 34 years of age with a six-month history of pain in the left testicle and thickening of the left spermatic cord, who developed swelling of the ankles and heavy proteinuria. He was thought to have a renal vein thrombosis producing the nephrotic syndrome and a left-sided varicocoele, but renal biopsy showed definite evidence of Ellis type 2 
nephritis, with widespread capsular adhesions and thickening of the capillary walls within the glomerular tufts. In the other 27 cases, renal biopsy either confirmed the clinical diagnosis, or provided a histological answer to the question of aetiology of a chronic renal disease state, which could, perhaps justifiably, be considered academic.

Two patients with hypertension and renal failure who showed the changes of benign hypertensive nephrosclerosis were found at subsequent postmortem examination to have polycystic kidneys.

\section{COMPLICATIONS}

Examination of mid-stream specimens of urine from 20 patients two hours after renal biopsy showed an increase in red-cell excretion in 10 of 17 successful biopsies, compared with the pre-biopsy urine examination. In the three cases in which biopsy had been unsuccessful, there was no change in the postbiopsy urine specimen, as might be expected.

Slight blood-staining of the urine for a few hours was frequently seen, but haematuria persisted for 24 hours in only two patients and then completely resolved. Evidence of a perirenal haematoma, with tenderness and bruising in the loin after biopsy, was seen in one patient. Soreness in the loin for a few hours was noted by a few patients only and was severe in one.

There were no other complications noted after renal biopsy in these cases.

\section{DISCUSSION}

The value of any aid to diagnosis may be judged by the ease with which it can be carried out and by the amount of information that it supplies.

Biopsy of the liver with a Menghini needle is a relatively simple procedure, and we have obtained adequate specimens in more than 200 patients, without a single failure or serious complication. Renal biopsy is more difficult however. Kerr (1960) pointed out that the kidney is not an easy target, that the specimens obtained are not always histologically adequate, and that although dangerous complications are rare, troublesome ones are fairly common.

Our experience suggests that the first two of these are the most important. The kidney is a mobile structure and, especially when small and fibrotic, may move forwards in front of the biopsy needle, which may therefore not gain access to renal tissue. The problem of adequacy is more complex. Some authors (Muehrcke, Kark, and Pirani, 1955; Phillippi, Robinson, and Langelier, 1961) claim that five or more glomeruli should be adequate for pathological diagnosis in diffuse lesions. However, Kellow,
Cotsonas, Chomet, and Zimmerman (1959) evalua- $\frac{\mathrm{o}}{.}$ ted the adequacy of needle-biopsy specimens of the $\overrightarrow{\vec{F}}$ kidney by comparing such specimens obtained at necropsy with the gross pathological findings in 103 cases. They found that $76 \%$ accurately reflected the $\frac{\bar{\sigma}}{\bar{c}}$ primary histological abnormality, and in only $69 \% \vec{\nabla}$ could an accurate diagnosis be made. In general, $\varrho$ diffuse rather than local diseases were diagnosed क accurately, as might be expected. These authors $\overrightarrow{0}$ point out the fallacy of relating representativeness to $\overrightarrow{-}$ the number of glomeruli; sometimes a diagnosis can- $\vec{\omega}$ not be made even when many are present, while, on the other hand, one glomerulus may be sufficient to? suggest or confirm a diagnosis. This would agree with $\vec{\sigma}$ our experience, in which a diagnosis could be made $i$ in three patients on the basis of one glomerulus in $\underset{\infty}{+}$ each of their biopsy specimens, while in three patients with more than 10 glomeruli, no diagnosis could be made.

Ross and Ross (1957) considered that careful $\mathbb{D}$ clinical assessment together with laboratory and $\mathbb{D}$ radiological information could allow a diagnosis to $\frac{3}{\sigma}$ be made in most cases of glomerulonephritis and $\stackrel{\mathbb{C}}{-}$ renal biopsy only provides confirmation. They found $\vec{\bullet}$ that the differentiation between benign and malig- of nant hypertension and the presence of pyelonephritis could not always be made with certainty by renal biopsy, and that its most useful role lay in the differential diagnosis of the nephrotic syndrome. Joekes, Heptinstall, and Porter (1958) studied 20 patients $\frac{}{\mathbb{D}}$ with the nephrotic syndrome and claimed that there was no consistent relationship between clinical fea- $\overline{\bar{O}}$ tures and histological abnormalities, and therefore renal biopsy was essential in order to decide what form of treatment to adopt and enable a prognosis to be made in some cases. We would agree that biopsy often provides a histological basis for patients $:$ with this condition but in four of 11 patients in the present series no abnormality was found in the renal biopsy with light microscopy. It is very probable that $₹$ electron microscopy would reveal abnormalities, but $\mathrm{O}$ an electron microscope is not part of the equipment of a general hospital. In one of these patients rectalo biopsy showed amyloidosis, although the renal $\stackrel{\sim}{\sim}$ biopsy specimen was negative for amyloid. Five of ? the patients with hypertension were considered $N$ clinically to be in the 'malignant' phase, but none of N them showed evidence of fibrinoid necrosis in the 0 biopsy specimens. This seemed surprising but is in agreement with the findings of Kincaid-Smith, $\frac{\mathscr{\Phi}}{\mathscr{C}}$ McMichael, and Murphy (1958), who showed in $\stackrel{0}{+}$ necropsy material that the vascular and parenchymal 0 lesions characteristic of malignant nephrosclerosis 0 궁 represent the final stages of malignant hypertension, $\vec{\otimes}$ and that if death from another cause preceded renalष्ठ failure, these lesions were slight or absent. 
In two other patients who were investigated for hypertension, the only significant changes found were those of benign nephrosclerosis. Subsequently at post-mortem examination they were found to have polycystic disease of the kidneys, which had not been demonstrable radiologically during life. It is evident that such a diagnosis would be difficult to make on the basis of a needle biopsy specimen.

Kark, Muehrcke, Pollak, Pirani, and Kiefer (1958), on the basis of 500 biopsies using the VimSilverman needle, claimed that culture of the specimen could often provide accurate bacteriological information if infection was present. This has not been confirmed in general experience, however. Jacobson and Newman (1962) found that the biopsy specimen was sterile in more than $90 \%$ of cases showing histological evidence of inflammation. We attempted to culture part of the biopsy specimen in the earlier cases of this series, but the technical problems involved, and the consistent lack of information derived, deterred us from continuing with the procedure.

Many authors stress the importance of renal biopsy in the differential diagnosis of anuria, especially in selecting patients suitable for dialysis. This is undoubtedly true, but in these cases it is surely better for the biopsy to be performed in the unit where the decision as to dialysis must be made, rather than in the general hospital from which the patient was referred. Frozen sections may be necessary, and the skill in preparing and examining such specimens is usually limited to special units. It has, therefore, been our practice to transfer anuric patients to a dialysis unit without renal biopsy rather than waste valuable time by carrying out the procedure ourselves.

It is difficult to be certain why the biopsy procedure failed in some patients. One was unconscious at the time of biopsy, and post-mortem examination demonstrated small hard kidneys with acute or chronic pyelonephritis, and it is not surprising that the needle failed to penetrate. In three patients, plain radiographs or intravenous pyelograms had shown the kidneys to be normal in size and position, while in another three patients renal outlines were not visualized and intravenous pyelography was not possible because of impaired renal function. In one of the patients, chronic glomerulonephritis was sub- sequently demonstrated at post-mortem examination and it is probable that some of the others also had firm, contracted kidneys. This is not, however, to remove completely the blame for failure from faulty technique.

Kark et al. (1958) were enthusiastic in advocating the value of renal biopsy. 'We are convinced that each biopsy specimen we examine profoundly affects the management of the illness, while the tissue harvested by percutaneous biopsy provides the most significant evidence on which to forecast the course of a disease involving the kidneys'. There are other authors, however, who are less willing to adopt renal biopsy as a routine laboratory procedure (Leadbetter 1958; Earle and Jennings, 1960; Litman, Yuile, Latta, Glicklich, and Smith, 1961). While there is no doubt that this may be a valuable procedure in the renal unit of a teaching hospital where there are facilities for electron microscopy and dialysis, and where medical staff are available to study serial sections throughout a biopsy specimen, one would hesitate to claim as much for it in a busy general hospital, where careful clinical and laboratory assessment provides a reasonable diagnosis in most cases and a biopsy is usually only confirmatory.

I thank Dr. G. S. C. Sowry for his help in preparing this paper, and Drs. J. Hamilton-Paterson and W. Brumfitt for their histological reports.

\section{REFERENCES}

Brun, C. (1954). Acute Anuria. Munksgaard, Copenhagen.

Earle, D. P., and Jennings, R. B. (1960). Med. Clin. N. Amer., 44, 59. Iversen, P., and Brun, C. (1951). Amer. J. Med., 11, 324.

Jacobson, M. H., and Newman, W. (1962). Arch. intern Med., 110, 211.

Joekes, A. M., Heptinstall, R. H., and Porter, K. A. (1958). Quart. J. Med., 27, 495.

Kark, R. M., and Muehrcke, R. C. (1954). Lancet, 1, 1047. , Pollak, V. E., Pirani, C. L., and Kiefer, J. H. (1958). Arch. intern. Med., 101, 439.

Kellow, W. F., Cotsonas, N. J., Chomet, B., and Zimmerman, H. J. (1959). Ibid., 104, 353.

Kerr, D. N. S. (1960). Lancet, 2, 1370.

Kincaid-Smith, P., McMichael, J., and Murphy, E. A. (1958). Quart. J. Med., 27, 117.

Leadbetter, W. F. (1958). Ann. Rev. Med., 9, 141

Litman, N. N., Yuile, C. L., Latta, H., Glicklich, D., and Smith, F. G. (1961). Amer. J. Dis. Child., 102, 321.

Muehrcke, R. C., Kark, R. M., and Pirani, C. L. (1955). New. Engl. J. Med., 253, 537.

Parrish, A. E., and Howe, J. S. (1953). J. Lab. clin. Med., 42, 152.

Phillippi, P. J., Robinson, R. R., and Langelier, P. R. (1961). Arch. intern. Med., 108, 739.

Ross, J. H., and Ross, I. P. (1957). Lancet, 2, 559. 